\title{
Influência da Temperatura e do Tipo de Substrato na Produção de Larvas de Musca domestica Linnaeus, 1758 (Diptera, Muscidae) ${ }^{1}$
}

\author{
Stefan Cruz Weigert ${ }^{2}$, Mario Roberto Chim Figueiredo ${ }^{3}$, Daniel Loebmann ${ }^{4}$, \\ José Augusto Reis Nunes ${ }^{5}$, Antonio Luís Garcia dos Santos ${ }^{6}$
}

\begin{abstract}
RESUMO - Em sala climatizada no Ranário Experimental da Fundação Universidade Federal do Rio Grande (REURG), quatro moscários foram preparados, colocando-se em cada um cerca de 5000 pupas de Musca domestica. Após o nascimento das moscas, cada moscário recebeu, além das bandejas com alimento (açúcar e leite), uma bandeja com substrato para postura, constituído de farelo de trigo umedecido. Diariamente os substratos para postura foram homogeneizados e distribuídos entre 15 bandejas pequenas, acondicionadas em estufas climatizadas e submetidos a uma combinação de cinco temperaturas $\left(20,23,26,29\right.$ e $\left.32^{\circ} \mathrm{C}\right)$ e três substratos para a produção de larvas (farelos de arroz, de trigo e de soja). As temperaturas de 20,23 e $26^{\circ} \mathrm{C}$ proporcionaram os melhores resultados de produção de larvas, a qual diminuiu com a elevação da temperatura, indicando ser desnecessária a utilização de aquecimento no larvário em locais onde a temperatura mínima não seja inferior a $20^{\circ} \mathrm{C}$. O farelo de trigo foi o melhor substrato para a produção de larvas de M. domestica. A maior produtividade de larvas foi verificada na $7^{\mathrm{a}}$ e $8^{\mathrm{a}}$ posturas.
\end{abstract}

Palavras-chave: larvas de mosca, ranicultura, substrato, temperatura

\section{Effect of Temperature and Kind of Substratum on the Production of Musca domestica Linnaeus, 1758 (Diptera, Muscidae) Larvae}

\begin{abstract}
In room acclimatized in Experimental Frog-Farm of Fundação Universidade Federal of Rio Grande (REURG) four flies cages were prepared, being put in each one about 5000 pupas of Musca domestica. After the birth of the flies, each flie cage received, above the trays with food (sugar and milk), a tray with substratum for posture, constituted of humidified wheat flour. Daily the substratum for posture were homogenized and distributed among 15 small trays, conditioned in acclimatized stoves and submitted to a combination of five temperatures $\left(20,23,26,29\right.$ and $\left.32^{\circ} \mathrm{C}\right)$ and three substratum for the production of larvae (flours of rice, wheat and soy). The temperatures of 20,23 and $26^{\circ} \mathrm{C}$ provided the best results of production of larvae, that decreased as temperature increased, indicating to be unnecessary the heating use in the larvae cages in places where the minimum temperature is not lower than $20^{\circ} \mathrm{C}$. The wheat flour went the best substratum to the production of larvae of $M$. domestica. The largest productivity of larvae was verified in the $7^{\text {th }}$ and $8^{\text {th }}$ postures.
\end{abstract}

Key Words: fly larvae, frog culture, substratum, temperature

\section{Introdução}

O ciclo vital da mosca doméstica, Musca domestica Linnaeus, 1758 (Diptera, Muscidae), compreende as fases de ovo, larva, pupa e mosca adulta. O estudo das formas de criação destes animais tornase fundamental em vista da possibilidade de sua utilização na alimentação animal, com principal atenção à fase larval.

Pro et al. (1999) afirmaram que a larva da M. domestica apresenta várias características que a torna adequada para uma produção massiva, como a precocidade e prolificidade da espécie, dependendo da temperatura e umidade ambientais. Uma fêmea é capaz de realizar desovas com até 800 ovos (Tinsley et al., 1984, citados por Pro et al., 1999). Além disso, a larva pode desenvolver-se em uma grande variedade de substratos, alimentando-se basicamente de matéria orgânica vegetal (Aleixo et al., 1984).

Lima \& Agostinho (1984) apresentaram a primeira proposta de se utilizar ração balanceada como alimento para rãs. Desde então, larvas de $M$. domestica vêm sendo utilizadas como artifício para estimular a ingestão da ração (Aleixo et al., 1984), pois as

\footnotetext{
1 Projeto apoiado pelo CNPq e pela FAPERGS, com auxílio e bolsas.

${ }^{2}$ Oceanólogo, bolsista de aperfeiçoamento da FAPERGS. E.mail: weigerts@bol.com.br

3 Prof. Adj. IV, FURG, DOc, LAC, REURG. C.P. 474, CEP: 96201-900, Rio Grande, RS. Orientador. E.mail: docchim@furg.br

4 Oceanólogo, estudante de mestrado em Oceanografia Biológica, FURG. E.mail: ocedl@furg.br

${ }^{5}$ Médico Veterinário, MSc em Engenharia de Alimentos, FURG. E.mail: augustoreisnunes@bol.com.br

${ }^{6}$ Oceanólogo, bolsista de iniciação científica do CNPq. E.mail: algsoceano@yahoo.com.br
} 
mesmas possuem fotofobia e imergem na ração, movimentando-a. Como as rãs, na fase terrestre, são essencialmente carnívoras, o movimento das larvas é fundamental para a sua alimentação (Reeder, 1964).

De acordo com Pro et al. (1999), o alto conteúdo de energia metabolizável das larvas secas, para frangos de corte (Energia Metabolizável aparente $=4071$ $\pm 133 \mathrm{kcal} / \mathrm{kg}$ ), pode ser explicado por seu alto conteúdo de óleo $(22 \%)$ e pela alta proporção de ácidos graxos insaturados (aproximadamente $49 \%$ do total), o que melhora a absorção dos ácidos graxos saturados.

Com o objetivo de otimizar a produção de larvas de $M$. domestica, foram realizados experimentos combinando-se diferentes temperaturas e substratos para criação dessas larvas, a fim de determinar a faixa de temperatura mais adequada no interior do larvário, bem como o substrato que proporciona a maior produção de larvas.

\section{Material e Métodos}

O experimento foi realizado no Ranário Experimental da Fundação Universidade Federal do Rio Grande (REURG), localizado no município do Rio Grande, RS. Para a realização do experimento foram utilizadas duas salas: uma sala climatizada que abrigava quatro moscários e outra equipada com cinco estufas climatizadas.

Os moscários são estruturas medindo 0,65 x 0,60 x 0,55 m (C x L x A), compostas de armação de madeira recoberta por tela de náilon branca com diâmetro de $1,0 \mathrm{~mm}$, com uma pequena porta frontal, de compensado, por onde é realizado o manejo diário. A sala onde foram instalados os quatro moscários utilizados no experimento foi especialmente preparada para este fim, sendo climatizada através de duas resistências elétricas de $500 \mathrm{~W}, 220 \mathrm{~V}$, controladas por um termostato. Durante o período experimental, a temperatura foi monitorada diariamente com o auxílio de um termômetro de máximas e mínimas, sendo que a temperatura média foi de $27^{\circ} \mathrm{C}$, com um desvio padrão de $2^{\circ} \mathrm{C}$. Esta sala contava com ventilação e iluminação naturais, realizadas por uma janela basculante. As cinco estufas climatizadas foram descritas por Figueiredo (1996) e Figueiredo et al. (2001), sendo modificadas pela retirada das gaiolas de fibra de vidro, as quais não foram utilizadas neste experimento.

Para o experimento quatro moscários foram preparados, lavando-se os mesmos com água hiperclorada e colocando-se em cada um cerca de 5.000 pupas de moscas da espécie Musca domestica, provenientes de larvas produzidas anteriormente nos próprios moscários.

Após o nascimento, as moscas foram alimentadas com açúcar, em uma bandeja, e leite misturado com água, na proporção de 1:1 (aproximadamente $25 \mathrm{~mL}$ de cada), em outra bandeja. A mistura leite/água foi trocada diariamente e coberta com uma camada de papel absorvente para evitar o afogamento das moscas.

Cinco dias após o nascimento das moscas, cada moscário recebeu o substrato para postura dos ovos. No preparo deste substrato utilizaram-se $400 \mathrm{~g}$ de farelo de trigo e $400 \mathrm{~mL}$ de água, que foram misturados em uma bandeja pequena até formar uma massa homogênea, onde se faziam furos com os dedos.

Diariamente, durante 16 dias (repetições), substituíam-se os substratos de postura de todos os moscários. Os quatro substratos retirados foram homogeneizados para evitar a formação de placas e permaneceram sobre os moscários até o dia seguinte, quando então, foram misturados em bandeja grande $(13 \mathrm{~L})$, previamente tarada. Esta mistura foi pesada, e o peso obtido dividido em 15 parcelas iguais, colocando-se em 15 bandejas pequenas (de 2,2 L), as quais foram distribuídas em uma combinação de três substratos para a produção de larvas (farelo de trigo - FT, farelo de arroz - FA e farelo de soja - FS) com cinco temperaturas $\left(20,23,26,29\right.$ e $\left.32^{\circ} \mathrm{C}\right)$.

Os substratos para produção de larvas foram preparados da mesma forma que o substrato de postura, apenas substituindo-se o farelo de trigo por farelo de arroz e soja, compondo-se três lotes de cinco bandejas, um para cada substrato (FT, FA e FS). Depois de prontos os substratos foram adicionados às 15 bandejas, até então com o farelo de trigo e larvas, sendo estas acondicionadas nas estufas climatizadas.

Cada bandeja permaneceu por três dias na estufa. Após este período, foi realizada a separação total das larvas do substrato, de cada bandeja separadamente, com auxílio de peneiras e feita a sua pesagem. Para a pesagem das amostras foi utilizada balança eletrônica com sensibilidade de $0,1 \mathrm{~g}$.

Para a determinação do ciclo produtivo de um moscário, os pesos de larvas das 15 bandejas foram somados e a soma dividida por quatro, obtendondo-se a produção média diária por moscário, submetendo-se, então, os resultados à análise de regressão.

Os dados foram submetidos à analise de variância e a testes de comparação de médias, sendo utilizado o software SAEG (Euclides, 1983), e a uma análise de regressão.

\section{R. Bras. Zootec., v.31, n.5, p.1886-1889, 2002}




\section{Resultados e Discussão}

A temperatura e o substrato de produção afetaram significativamente $(\mathrm{P} \leq 0,05)$ a produção de larvas de $M$. domestica, não sendo significativo o efeito da interação entre ambos fatores em questão.

Verificou-se maior produção de larvas a 20,23 e $26^{\circ} \mathrm{C}$ do que a 29 e $32^{\circ} \mathrm{C}$. Com relação ao substrato utilizado, o farelo de trigo foi melhor que farelo de arroz e soja (Tabela 1).

A distribuição dos pesos médios de larvas, obtidos em função da temperatura das estufas, ajusta-se a um modelo linear (Figura 1), no qual se observa decréscimo da produtividade de larvas com o aumento da temperatura ambiente.

Observou-se que temperaturas mais elevadas (29 e $32^{\circ} \mathrm{C}$ ) afetaram negativamente a produção de larvas
M. domestica. O processo de fermentação do substrato de produção, que ocorre durante o desenvolvimento das larvas, caracteriza-se por ser exotérmico, ou seja, durante a fermentação ocorre liberação de calor.

A Figura 2 ilustra a produção média de larvas em função do substrato de produção utilizado. Nela se observa que o maior peso de larvas foi obtido com farelo de trigo.

A produção de larvas é viável até 21 dias após o nascimento das moscas, ou seja, durante 16 dias a partir da primeira postura (Figura 3). A dispersão dos dados de peso médio de larvas (y) ao longo do tempo (x), ficou bem caracterizada por um modelo quadrático (Figura 3), no qual se observou que a máxima produtividade do moscário ocorreu entre o $7^{\circ}$ e $8^{\circ}$ dia após o início das posturas, ou seja, entre o $12^{\circ}$ e $13^{\circ}$ dia após o nascimento das moscas. Este resultado comprova

Tabela 1 - Peso médio de larvas (g) em função da temperatura do larvário e do substrato de produção utilizado Table 1 - Larvae' average weight $(g)$ as a function of the temperature of the larvae cage and of the used production substratum

\begin{tabular}{lccc}
\hline $\begin{array}{l}\text { Temperatura }\left({ }^{\circ} \mathrm{C}\right) \\
\text { Temperature }\left({ }^{\circ} \mathrm{C}\right)\end{array}$ & $\begin{array}{c}\text { Peso médio de larvas }(\mathrm{g}) \\
\text { Larvae average weight }(\mathrm{g})\end{array}$ & $\begin{array}{c}\text { Substrato de produção } \\
\text { Production substratum }\end{array}$ & $\begin{array}{c}\text { Peso médio de larvas }(\mathrm{g}) \\
\text { Larvae average weight }(g)\end{array}$ \\
\hline 20 & $45,72^{\mathrm{a}}$ & & $44,88^{\mathrm{a}}$ \\
23 & $41,71^{\mathrm{a}}$ & Farelo de Trigo & $32,83^{\mathrm{b}}$ \\
26 & $40,90^{\mathrm{a}}$ & Farelo de Soja & $32,58^{\mathrm{b}}$ \\
29 & $30,59^{\mathrm{b}}$ & Farelo de Arroz & \\
32 & $24,90^{\mathrm{b}}$ & &
\end{tabular}

Médias, na coluna, seguidas de letras diferentes, diferem $(P \leq 0,05)$ pelo teste de Newman Keuls.

Means, in a column, followed by different letters, differ $(P \leq .05)$ by Newman-Keuls test.

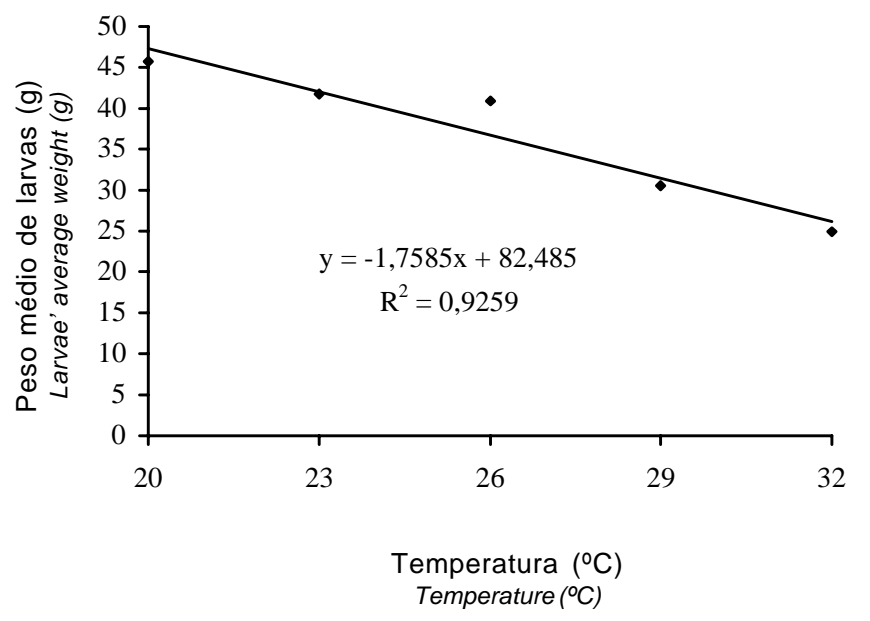

Figura 1 - Estimativa do peso de larvas em função da temperatura da estufa.

Figure 1 - Estimate of larvae weight as a function of the stove temperature.

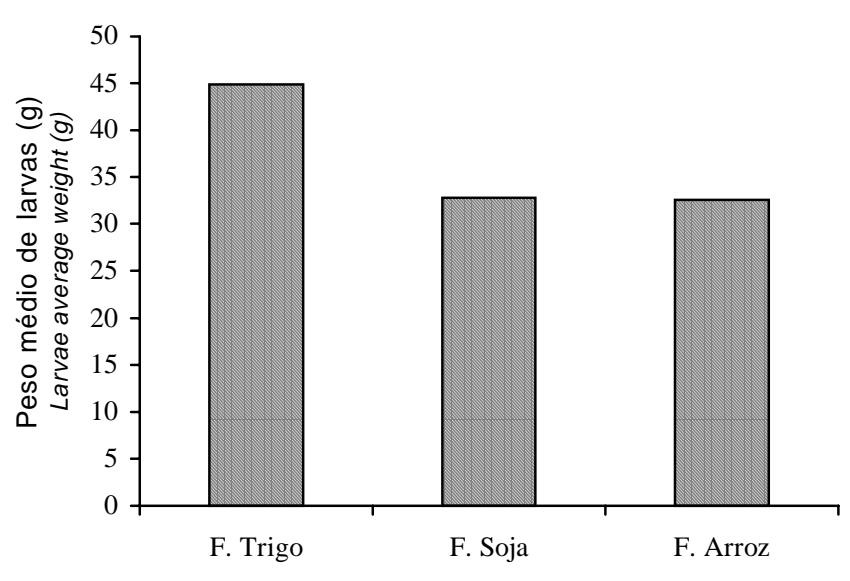

Substrato de produção Production substratum

Figura 2 - Peso de larvas em função do substrato de produção utilizado.

Figure 2 - Larvae weight as a function of the used production substratum.

R. Bras. Zootec., v.31, n.5, p.1886-1889, 2002 
que a periodicidade média para a montagem de moscários, tendo em vista a necessidade de se manter estável a produção de larvas, é de sete dias, sendo necessários, no mínimo, quatro moscários para o rodízio, conforme sugerido por Aleixo et al. (1984).

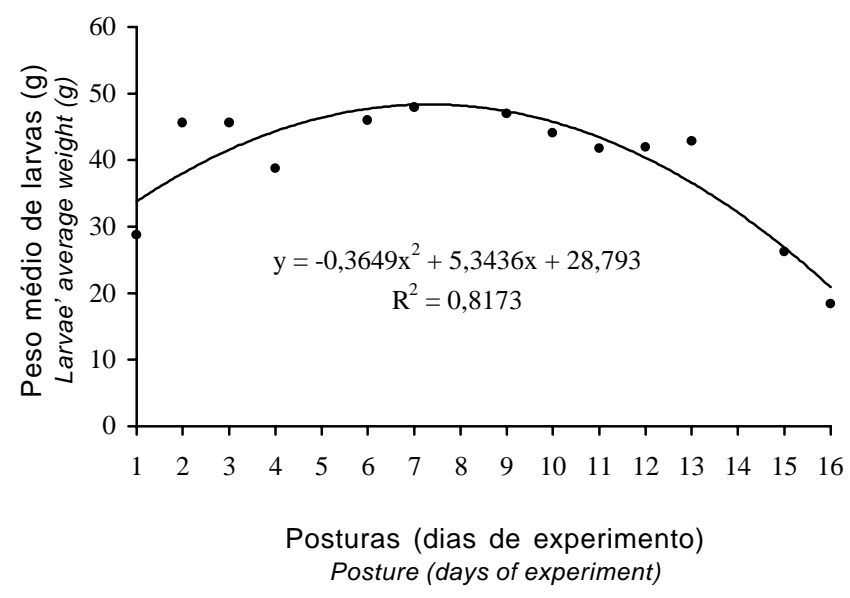

Figura 3 - Estimativa dos pesos médios de larvas ao longo da postura das moscas.

Figure 3 - Estimate of larvae average weight during the flies posture.

\section{Conclusões}

A produção de larvas é inversamente proporcional à elevação da temperatura, não sendo necessária a utilização de aquecimento no larvário (o que representa uma economia para o produtor).

Entre os farelos utilizados como substrato de produção, além da maior produção de larvas, é indiscutível a facilidade de manejo (umedecimento e separação de larvas) proporcionada pelo farelo de trigo.

A máxima produtividade de um moscário ocorre entre a $7^{\mathrm{a}}$ e $8^{\mathrm{a}}$ posturas, de modo que, nesses dias deve ser realizada a retirada de larvas para a montagem de novo(s) moscário(s).

Os resultados sugerem a necessidade de novos experimentos a partir de temperaturas abaixo de $20^{\circ} \mathrm{C}$.

\section{Literatura Citada}

ALEIXO, R.C.; LIMA, S.L.; AGOSTINHO, C.A. Criação da mosca doméstica para suplementação alimentar de rãs. Viçosa, MG: Universidade Federal de Viçosa, 1984. 11 p. (Informe Técnico, 46).

EUCLIDES, R.F. Sistema para análises estatísticas e genéticas. Manual de utilização do SAEG. Viçosa, MG: Universidade Federal de Viçosa, 1983. 57p.

FIGUEIREDO, M.R. Influência dos fatores ambientais sobre o desempenho da rã-touro (Rana catesbeiana Shaw, 1802) em gaiolas. Viçosa, MG: Universidade Federal de Viçosa, 1996. 151p. Tese (Doutorado em Zootecnia) - Universidade Federal de Viçosa, 1996.

FIGUEIREDO, M.R.C.; LIMA, S.L., AGOSTINHO, C.A. et al. Estufas climatizadas para experimentos ambientais com rãs, em gaiolas. Revista Brasileira de Zootecnia, v.30, n.4, p.1135-1142, 2001.

LIMA, S.L.; AGOSTINHO, C.A. Técnicas e propostas para alimentação de rãs. Viçosa, MG: Universidade Federal de Viçosa, 1984. 11p. (Informe técnico 50).

PRO, A.M.; CUCA, M.G.; BECERRIL, C.P. et al. Estimación de la energía metabolizable y utilización de larva de mosca (Musca domestica L.) en la alimentación de pollos de engorda. Archivos Latinoamericanos de Producción Animal, v.7, n.1, p.39-51, 1999.

REEDER, W.G. The digestive system. In: MOORE, J.A. (Ed.) Physiology of the Amphibia. New York: Academic Press, 1964. v.1, p.99-209.

Recebido em: 30/11/01 Aceito em: 07/06/02 